\title{
Friction of a slider on a granular layer: Non-monotonic thickness dependence and effect of boundary conditions
}

\author{
Saloome Siavoshi, Ashish V. Orpe and Arshad Kudrolli \\ Department of Physics, Clark University, Worcester, Massachusetts 01610
}

(Dated: June 19, 2018)

\begin{abstract}
We investigate the effective friction encountered by a mass sliding on a granular layer as a function of bed thickness and boundary roughness conditions. The observed friction has minima for a small number of layers before it increases and saturates to a value which depends on the roughness of the sliding surface. We use an index-matched interstitial liquid to probe the internal motion of the grains with fluorescence imaging in a regime where the liquid has no significant effect on the measured friction. The shear profiles obtained as a function of depth show decrease in slip near the sliding surface as the layer thickness is increased. We propose that the friction depends on the degree of grain confinement relative to the sliding surfaces.
\end{abstract}

PACS numbers: $45.70 . \mathrm{Ht}, 45.70 . \mathrm{Mg}$

The friction encountered by a mass sliding on a thin granular layer is important in a variety of contexts such as walking on sand, braking on a pebble strewn road, and jamming of joints and bearings in a dusty environment. Such systems consists of two linearly sheared surfaces with a granular layer in between. Assuming for simplicity that the material properties of the grains and the surfaces are the same, a basic issue one would like to understand is how the granular case differs from when solid surfaces slide past each other. In particular one would like to know the magnitude of the friction as a function of layer thickness and the roughness of the boundaries.

A number of studies have examined shear of deep granular layers with linear, couette, and drag systems 1, 2, 3, 4, 5, 6, 7, 8, 9]. A shear zone confined over a few grain diameters near one of the boundaries is usually observed. Qualitative difference between granular and solid-on-solid stick-slip friction have been noted due to dilatancy [3, 10, 11, 12]. The friction coefficient and the dilatancy of the shearing layer has been found to be independent of the shearing rates for deep layers [6]. Nonetheless, measurements which span the range from solid-on-solid to granular friction have not been accomplished and analyzed in any detail. In experiments with granular flows down rough planes 14], the inclination required to have steady motion is observed to decrease with an increase in the layer thickness, and thus friction may be interpreted as decreasing with increasing granular layer thickness. However, the material is free to expand at the top surface which is a crucial difference.

Here, we report the sliding friction of a mass on a thin granular bed to address open questions in confined and sheared granular matter. The friction of the slider with a rough surface moving on a rough substrate decreases sharply as a grain layer is added, before increasing and saturating as the bed thickness is increased over ten layers. Exploiting the fact that the behavior remains unchanged at low shear rates in the presence of an interstitial liquid, we use an index-matching technique to probe

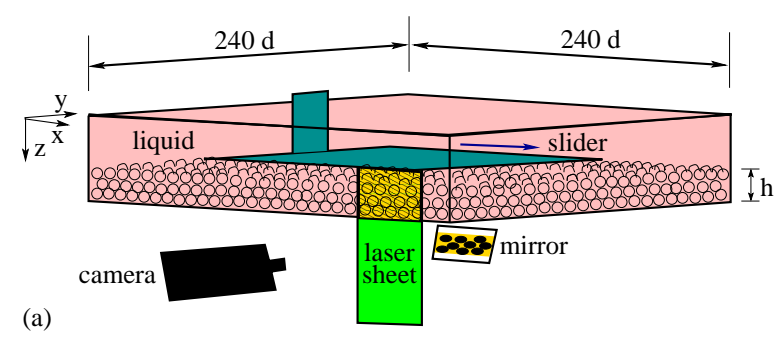

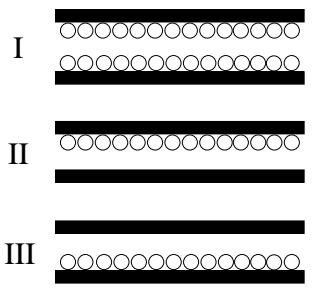

(b)

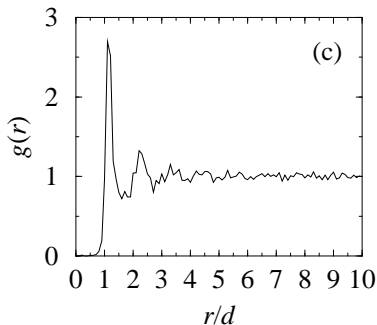

r/d
FIG. 1: (Color Online) (a) Schematic of experimental apparatus. (b) Three kinds of boundary surface conditions I: rough-on-rough, II: rough-on-smooth, and III: smooth-onrough. Rough surfaces are fabricated by gluing a layer of glass beads to the surfaces. (c) Two-point cross correlation function $g(r)$ as a function of distance of separation $r$ of two beads on the surface. Peaks at multiples of bead diameter are observed indicating absence of hexagonal packing.

the motion of the grains inside the bed. From these measurements, we propose that the change in the friction with layer thickness is because of the increased confinement and locking of a grain relative to its neighbors.

A schematic of the apparatus is shown in Fig. प(a). A rectangular slider of size $100 \mathrm{~mm} \times 140 \mathrm{~mm}$ and mass $m=0.146 \mathrm{~kg}$ is pushed over a granular layer with a linear translating stage connected to a stepper motor and is similar in design to that in Ref. [3]. The sliding plate is free to move vertically, and the experiment is carried out at constant pressure given by the weight divided by surface area. A stiff spring with spring con- 
stant $k=1.62 \times 10^{4} \mathrm{~N} \mathrm{~m}^{-1}$ is used to couple the slider to the translating stage and measure the force required to move the slider with the help of a capacitance displacement sensor. All the grains (of diameter $d=1.0 \pm 0.1$ $\mathrm{mm}$ ) and surfaces used to measure the frictional properties are composed of soda lime glass. Three combinations of smooth and rough boundary conditions are used as illustrated in Fig. 1(b). An optically polished glass surface is used for the smooth case. A layer of beads is glued on the planar slider and substrate surfaces in order to obtain rough boundary conditions. The positions of the grains obtained from an image of the surface, and then characterized by the two-point cross-correlation function $g(r)$ [see Fig. 1(c)] shows no hexagonal order.

The granular bed with a height $h$ and surface area of $240 \mathrm{~mm} \times 240 \mathrm{~mm}$ is prepared by pouring and leveling the grains with a knife edge. In order to obtain consistent initial conditions for the granular bed, we first place the slider on the granular bed and push the slider over a distance of approximately $15 d$ to pre-shear the system. We then hold the slider for 5 seconds to have a well defined pre-aging condition for the contact surfaces. We then push the slider with various speeds $v_{p}$ over a distance of $15 d$ to obtain the spring displacement with a sampling rate of $1 \mathrm{kHz}$. The slider either performs stick-slip or continuous motion depending on the pushing speed and the ratio of $k$ and $m$ [3]. For simplicity, we focus on the continuous sliding regime. The effective coefficient of sliding friction $\mu_{e f f}$ is obtained by averaging the displacement measured over time and multiplying it with $k$ and dividing by the slider weight.

Figure 2(a) shows the measured $\mu_{\text {eff }}$ for a slider with a rough boundary conditions as a function of $h / d$. A non-monotonic thickness dependence is observed with $\mu_{e f f}$ first decreasing rapidly as a layer of grains is added between the slider and the substrate. Then, $\mu_{e f f}$ increases and saturates as the number of layers are increased to about 10 . While one can imagine that friction between surfaces may decrease if grains are added due to a lubrication-like effect, the subsequent increase in friction with $h$ alerts us to the subtlety of the problem.

To understand the role of the boundary surfaces, $\mu_{\text {eff } f}$ for three different surface conditions [illustrated in Fig. [1 (b)] are plotted in Fig. 2(b). First, we focus on the case where the boundaries directly slide against each other $(h / d=0)$. As long as one of the surfaces is smooth, the measured value of $\mu_{e f f}$ is significantly lower than when both surfaces are rough. The value depends somewhat on the solvents used to clean the surfaces and relative humidity. Now the higher value for the rough surfaces can be understood by considering the geometry of the rough surfaces.

For simplicity consider that the rough surfaces can be represented by a row of beads next to each other as in Fig. 1(b). Then, depending on the relative angle $\theta$ from the vertical where grains on the two surfaces make con-
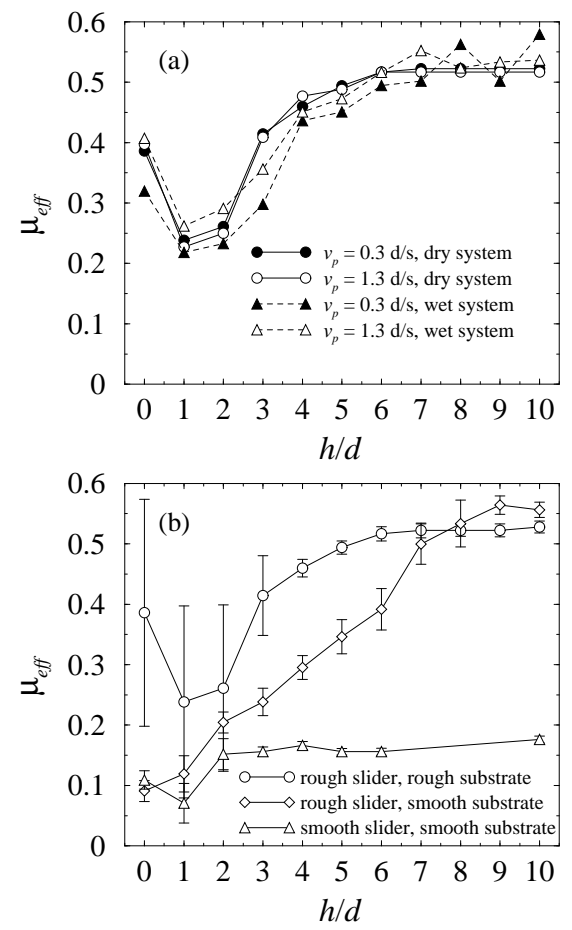

FIG. 2: (a) Effective coefficient of sliding friction $\left(\mu_{\text {eff }}\right)$ as a function of granular layer of thickness $h$ normalized by grain diameter $d$. All surfaces are rough. (b) $\mu_{e f f}$ for the case of dry system and three boundary conditions shown in Fig. 1(b). The measured fluctuations in friction are also shown. $\left(v_{p}=\right.$ $0.3 d / \mathrm{s})$

tact, the effective friction given by the ratio of the force required to see continuous motion and the weight of the slider can be shown to be $\tan \left(\theta_{0}+\theta\right)$, where $\mu_{0}\left(=\tan \theta_{0}\right)$ is the coefficient of friction for two smooth glass surfaces sliding against each other. Now, $\theta$ can vary between at least 0 and $\pi / 6$ depending on where neighboring beads touch each other, and therefore using the measured $\mu_{\text {eff }}$ for rough on smooth case for $\mu_{0}$ and using the average of the angles of the contact, one obtains the effective friction as 0.5 which is close but somewhat higher than what we measure for the rough on rough case. It is possible that a closer match may be obtained by using the actual distribution of contact angles.

Now let us examine the friction dependence on layer thickness. For a thick or deep enough granular layer, $\mu_{e f f}$ depends on the roughness of the sliding surface and does not depend on the nature of the substrate. As the number of layers is decreased, $\mu_{e f f}$ decreases except when the sliding surface is smooth in which case the friction encountered remains small and more or less constant [see Fig. [2(b)].

To obtain further insight into this problem, an understanding of the grain packing and velocity profiles inside the granular layer is necessary. A schematic of the setup used for the fluorescent imaging is also shown in 


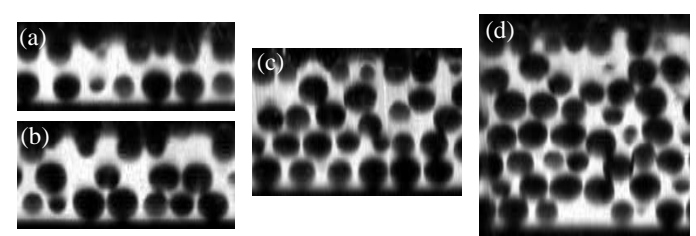

FIG. 3: Images of vertical slice of the granular bed away from the side boundaries for different layer thicknesses with roughon-smooth boundary conditions. (a) $h / d=1$, (b) $h / d=2$, (c) $h / d=4$, and (d) $h / d=7$.

Fig. 1(a). The grains are completely immersed in a liquid with a matching refractive index $(\approx 1.52)$. A fluorescent dye with excitation and emission frequencies centered at $525.5 \mathrm{~nm}$ and $565 \mathrm{~nm}$, respectively, is added to the liquid. As illustrated in Fig. 1(a), planes of the granular bed far from the side boundaries are illuminated using a $15 \mathrm{~mW}$ laser and cylindrical lens system and imaged with a digital camera. The internal imaging technique used is similar to that used in Ref. [15]. Typical images after rescaling and smoothing are shown in Fig. 3 for a few $h / d$. The apparent size of the beads depends on the distance of the bead center from the illumination plane. A centroid algorithm is used to find the particle position to sub-pixel resolution. Imaging at 30 frames per second is sufficient to track the particles and obtain mean velocities to within $5 \%$.

Figure 2(a) also shows $\mu_{\text {eff }}$ with the granular bed immersed in the liquid used for internal imaging. The obtained values are observed to be close to those for the dry case after correcting for the buoyant force due to the liquid displaced by the slider. Thus the measured values do not vary significantly at low shear rates. (However, the measured values depart systematically from the dry case if the slider speed is increased by an order of magnitude.)

Figure 4 shows the mean density normalized by the maximum packing density $\langle\rho\rangle /<\rho_{\max }>$ of the grains as a function of depth $z$ inside the bed for various layer thicknesses. Here, $z=0$ is taken to be the averaged lowermost points of the particles glued to the slider. Peaks are observed which get smaller and broader with increase in height. Thus significant layering is seen especially for lower heights independent of the overall thickness of the bed.

The corresponding mean velocity normalized by $v_{p}$ are plotted as a function of depth in Fig. 5 For one and for two layers, the slip almost entirely occurs between the slider and the granular layer. But as the granular layer thickness is increased, the slip region grows wider before saturating as the number of layers approaches 10 . As can be noted from Fig. 5( (e), the velocity profiles are more or less independent of the nature of the substrate for $h / d \sim 10$. We have fitted the asymptotic velocity profile with the following fit: $v / v_{p}=\exp \left(-a(h / d)-b(h / d)^{2}\right)$ where $a=0.6$, and $b=0.03$. Thus the form is mostly

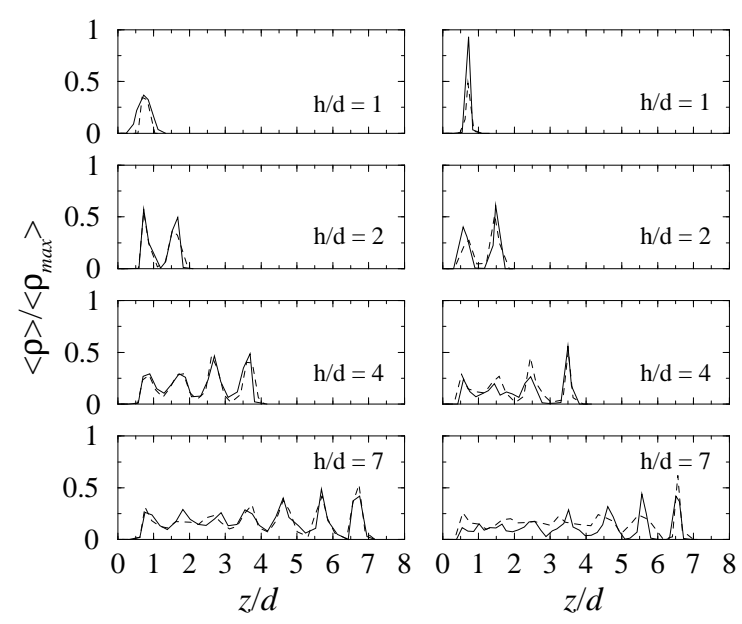

FIG. 4: (Color Online) Normalized density of the grains as a function of depth for the rough-on-rough (first column) and the rough-on-smooth (second column) boundary conditions and slider velocities $v_{p}=0.3 d / \mathrm{s}$ (solid lines) and $v_{p}=1.3 d / \mathrm{s}$ (dashed lines).

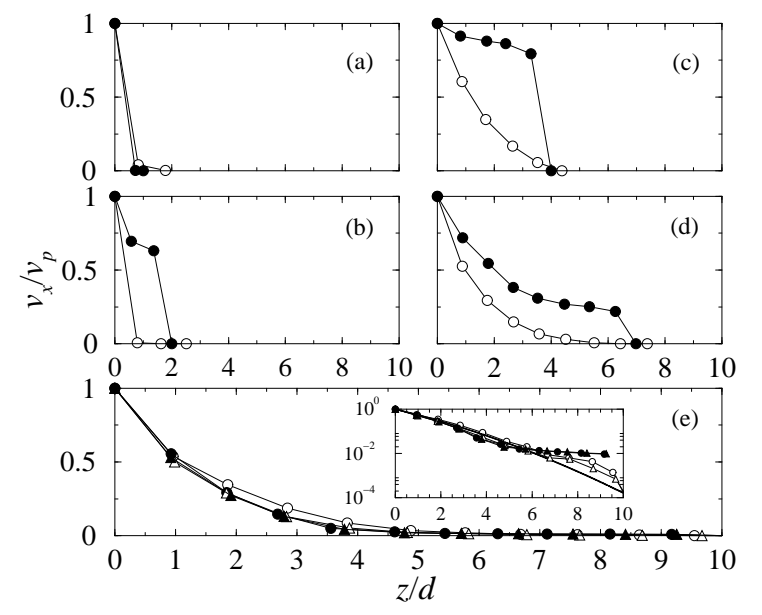

FIG. 5: Normalized mean velocity of the grains as a function of depth for different boundary conditions and slider velocities. (a)-(d) $h / d=1,2,4$, and 7 respectively and $v_{p}=0.3 d / s$. (e) $h / d=10, v_{p}=0.3 d / s(\circ), v_{p}=1.3 d / s(\Delta)$. Rough-onrough case (open symbols) and rough-on-smooth case (filled symbols). Inset: Corresponding plot in log-linear scale. The thick solid line represents the fit (see text).

exponential with a small correction similar to that obtained by Mueth et al [] in a couette geometry far away from side walls. A somewhat similar profile comprising of a linear part near the shearing surface followed by a slow exponential decay was obtained theoretically and numerically for 2D couette flow by Volfson, et al [17].

Having characterized the overall structure and velocity profiles, we next plot the vertical component of the trajectories of sample particles in the granular bed for various $h$ in order to understand the friction properties. 

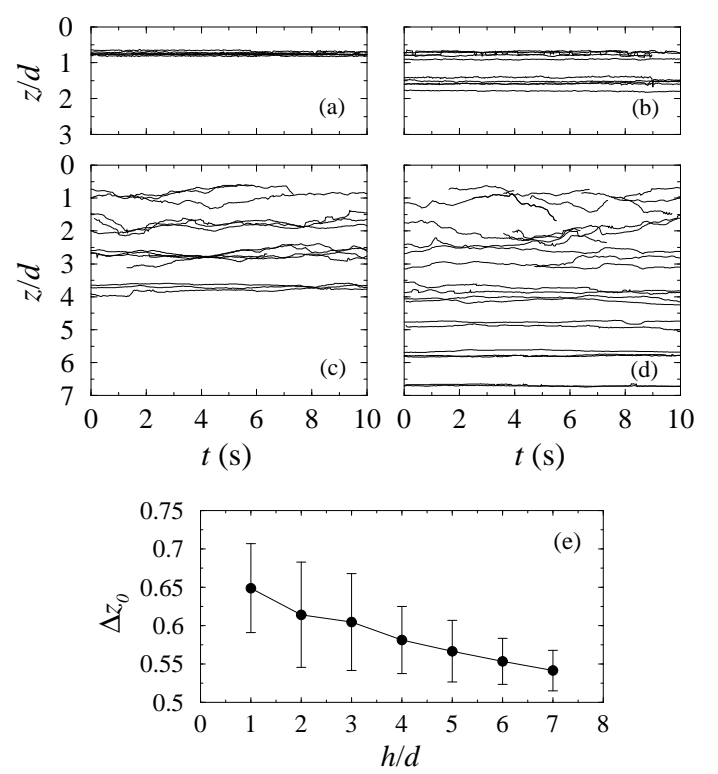

FIG. 6: Vertical component of the trajectory of a particle for different layer thicknesses and $v_{p}=0.3 d / s$. (a) $h / d=1$, (b) $h / d=2$, (c) $h / d=4$, and (d) $h / d=7$. Rough-onrough boundary conditions are used. (e) Average gap between sliding surface and top layer. The data is averaged over all boundary conditions and slider speeds.

As shown in Fig. 6] a,b), for $h / d=1$ and 2, the grains more or less remain at the same height, and do not exhibit significant mean drift [as can be noted from the velocity profiles plotted in Fig. [5 (a,b)]. From the movies of the grain motion [16], it may be easily noted that the particles more or less fluctuate in the same position. As $h$ is increased and the region of shear increases, grains can be seen to show increased motion in the vertical direction in addition to the translation motion along the direction of shear [see Fig. 6] (c,d) and 16]]. Because of the mobility of the grains in the shear zones, the layers rearrange so that the gap between the shearing surface and the granular layer below it decreases with $h$. To quantify this trend, we have measured the gap distance $\Delta z_{0}$ defined by the distance of the top layer of the particles in the bed from $z=0$ averaged over all images. The result is plotted in Fig. 6(e).

From these observations one can construct an argument for the increase and saturation of $\mu_{e f f}$ with $h / d$. Because of the greater gap between the shearing surface and the grains for small $h / d$, it can be noted that the grains are less confined. This allows grains to move around the bumps on the sliding surface more easily, which lowers the $\mu_{e f f}$. However, for greater $h / d$, the grains move until they are jammed against each other and the sliding surface, resulting in lower $\Delta z_{0}$. This causes the actual sliding surfaces between grains to be at angles other than normal to the horizontal, which results in greater $\mu_{\text {eff }}$ due to the additional contribution of the applied force to the normal force between sliding surfaces. Indeed, the measured value of $\mu_{e f f}$ for larger $h / d$ is similar to that discussed earlier for rough surfaces directly sliding past against each other.

Our explanation is also consistent with why an increase in friction is not observed in flows down inclined planes [2] because, a top confining surface does not exists in that case. While additional support for our argument could be given by examining particle-particle correlation functions within the layers, this is beyond the capability of our current technique.

In summary, we have examined the granular layer thickness dependence on the friction encountered by a mass sliding on a granular surface. The observed friction depends on the roughness of the sliding surface and for thin layers it depends on the roughness of the substrate as well. Friction is observed to increase with layer thickness. With the help of grain position data acquired using an index-matching technique, we give an explanation of the increase in friction in terms of the confinement and locking of the grains against its neighbours and the sliding surface. We have also shown how the shear profile changes with layer thickness and surface roughness. In these experiments, gravity clearly breaks the up-down symmetry. It would be interesting to consider how the phenomena will differ when this symmetry is not broken and will be the subject of future work.

We thank I. Nagle, R. O'Donnell and A. Samadani for help with acquiring preliminary data and construction of the apparatus. The work was supported by the National Science Foundation under Grant Nos. DMR-9983659 and CTS-0334587, and a DOE-GLUE grant and the GLUE program of the Department of Energy.

[1] P. A. Thompson and G. S. Grest, Phys. Rev. Lett. 67, 1751 (1991).

[2] G.D.R. MiDi, Eur. Phys. J. E 14, 341 (2004).

[3] S. Nasuno, A. Kudrolli, and J. P. Gollub, Phys. Rev. Lett. 79, 949 (1997); S. Nasuno, A. Kudrolli, A. Bak, and J. P. Gollub, Phys. Rev. E 58, 2161 (1998).

[4] W. Losert, J.-C. Géminard, S. Nasuno, and J. P. Gollub, Phys. Rev. E 61, 4060 (2000); J.-C. Géminard, W. Losert, and J. P. Gollub, Phys. Rev. E 59, 5881 (1999).

[5] K. Mair, K. M. Frye, and C. Marone, J. Geophys. Res. 107, 2219 (2002).

[6] C. Coste, Phys. Rev. E 70, 051302 (2004).

[7] D. M. Mueth, et al., Nature (London) 406, 385 (2000).

[8] I. Albert, et al., Phys. Rev. Lett. 84, 5122 (2000).

[9] J. Geng and R. P. Behringer, Phys. Rev. E 71, 011302 (2005).

[10] T. Baumberger, F. Heslot, and B. Perrin, Nature (London) 367, 544 (1994).

[11] H. Hayakawa, Phys. Rev. E 60, 4500 (1999).

[12] F. Lacome, S. Zapperi, and H. J. Herrmann, Eur. Phys. J. E 2, 181 (2000). 
[13] B. Francois, F. Lacombe, and H. J. Herrmann, Phys. Rev. E 65, 031311 (2002).

[14] O. Pouliquen, Phys. Fluids 11, 542 (1999).

[15] J.-C. Tsai, G. A. Voth, and J. P. Gollub, Phys. Rev. Lett. 91, 064301 (2003).
[16] Movies of grain motion for $h / d=1,2,4$, and 7 can be found at: http://physics.clarku.edu/ akudrolli/friction

[17] D. Volfson, L. Tsimring and I. Aranson, Phys. Rev. E, 69, 031302 (2004). 